\title{
Relationship between antibiotic susceptibility and biofilm production in a clinical strain of Burkholderia cepacia complex
}

\author{
Lucia Corich, Linda Furlanis, Francesco Gon, Fabrizia Gionechetti, Raffaela Bressan, \\ Lucilla Dolzani, Enrico Angelo Tonin, Cristina Lagatolla \\ Department of Life Sciences, University of Trieste
}

Key words: Burkholderia cepacia, biofilm, antibiotic resistance, MIC, BIC.

Relazione tra sensibilità agli antibiotici e produzione di biofilm in un ceppo clinico di Burkholderia cepacia complex

\section{SUMMARY}

Infections caused by Burkholderia cepacia complex in patients with cystic fibrosis (CF) are often related to increased mortality. One of the mechanisms that makes the bacteria more resistant to the action of antibiotics is the ability to produce biofilm. It has been compared the antibiotic resistance of two clinical isolates of a biofilm-producing strain called BTS-2, responsible for chronic pulmonary infection in a CF patient. Bacteria were incubated in both Yeast Extract Mannitol Medium (YEM) and Mueller Hinton Broth (MHB) at $30^{\circ} \mathrm{C}$ for 24 hours. Biofilm was quantified by spectrophotometer readings $\left(\mathrm{OD}_{570}\right)$ of the extracted crystal violet. By microdilution broth, we evaluated the minimum concentration of antibiotic inhibiting the growth of the planktonic form (MIC); by the technology "Calgary Biofilm Device" and by Resazurin, we evaluated the susceptibility of sessile forms (BIC). Data demonstrate that the microorganism in the course of time changed its sensitivity to some of the antibiotics tested. The comparison between sessile and planktonic forms of BTS2 pre-incubated in MHB showed that sessile forms increased their resistance to antibiotics in a few cases only. BICs of BTS2 pre-incubated in YEM, where the strain produced an amount of biofilm significantly lower than in MHB, showed a higher susceptibility to 4 of the 10 antibiotics tested. Our data showed that the susceptibility of sessile forms of a strain is not always lower than the susceptibility of its planktonic forms and that culture medium can affect significantly the biofilm production.

\section{INTRODUZIONE}

Le infezioni causate da Burkholderia cepacia complex (Bcc) in pazienti affetti da fibrosi cistica (FC) sono spesso correlate ad un aumento di mortalità e l'innata resistenza di questi microrganismi ad un ampio range di antibiotici complica il trattamento di tali pazienti.

Uno dei meccanismi che contribuisce a rendere $\mathrm{i}$ batteri più resistenti all'azione degli antibiotici è la capacità di produrre biofilm.

È stata paragonata la resistenza agli antibiotici da parte di due isolati indipendenti di un ceppo denominato BTS2, responsabile di un'infezione polmonare cronica in un paziente $\mathrm{FC}$.

I due isolati, prelevati a distanza di 6 anni (BTS200 e BTS2-06), mostravano una significativa differenza nella capacità di produrre biofilm se cresciuti in Yeast Extract Mannitol Medium (YEM) mentre tale capacità risultava paragonabile se $\mathrm{i}$ ceppi venivano fatti crescere in Mueller Hinton Broth (MHB).

\section{MATERIALI E METODI}

Valutazione in vitro della produzione di biofilm Il biofilm prodotto è stato quantificato come descritto (6), con 1'introduzione di alcune modifiche: $5 \times 10^{5}$ batteri sono stati inoculati nei pozzetti di una piastra microtiter e incubati per 24 ore a $30^{\circ} \mathrm{C}$. Dopo aver misurato il livello di crescita $\left(\mathrm{OD}_{590}\right)$, le colture sono state lavate per eliminare le cellule non adese, fissate con metanolo e il biofilm è stato colorato con cristalvioletto $2 \%$ e decolorato con acido acetico glaciale 33\%. Il biofilm prodotto è stato quantificato mediante lettura spettrofotometrica (OD 570) del colorante estratto. Sono stati effettuati almeno tre esperimenti indipendenti, ciascuno eseguito in triplicato. Le differenti quantità di biofilm sono state analizzate mediante Test t di Student; un valore di $p<0.05$ è stato considerato statisticamente significativo.

Valutazione della MIC e della BIC

Mediante microdiluizione in brodo (3) sono state valutate le minime concentrazioni di antibiotico in grado di inibire la crescita della forma planctoni-

\section{Corresponding author: Corich Lucia}

Università degli Studi di Trieste, Dipartimento di Scienze della Vita

Via L. Giorgieri, I - 34I00 TRIESTE - Tel.: 0405583693 - Fax: 040558369 I

E-mail: luxcor@yahoo.it 
ca di BTS2 (MIC); parallelamente, mediante l'utilizzo della tecnologia "Calgary Biofilm Device" $(2,4)$ nonché di un metodo che utilizza la resazurina come indicatore di vitalità cellulare (5), è stata valutata la suscettibilità delle forme sessili (BIC). Brevemente: i due isolati sono stati preincubati sia in MHB, ove la produzione di biofilm risultava pressochè la stessa, sia in YEM, ove la produzione di biofilm da parte di BTS2-00 risultava significativamente maggiore rispetto a quella di BTS2-06. Le forme sessili così ottenute sono state lavate e incubate in MHB addizionato con diluizioni seriali degli antibiotici elencati in Tabella 1.

\section{RISULTATI}

I valori di BIC ottenuti con le due tecniche descritte nei METODI sono risultati pressoché gli stessi e sono riportati, insieme alle corrispondenti MIC, in Tabella 1. Questi dati dimostrano che il microrganismo nel corso del tempo ha modificato la propria sensibilità ad alcuni degli antibiotici testati, probabilmente per l'acquisizione di determinanti di resistenza.

I dati ottenuti indicano che per metà dei farmaci testati (ceftazidime, ciprofloxacina, imipenem, levofloxacina e meropenem) il ceppo BTS2, preincubato in MHB, presenta valori simili di MIC e BIC. In particolare il ceftazidime, in accordo con dati già presenti in letteratura (1), risulta essere il farmaco più attivo nei confronti sia della forma planctonica che di quella sessile di BTS2.

L'associazione sulfametoxazolo/trimetoprim e la doxycyclina sembrano in grado di eradicare le forme sessili ma solo a concentrazioni molto elevate.

I rimanenti tre antibiotici mostrano un'attività buona (cefepime e piperacillina) o moderata (aztreonam) sulle forme planctoniche, ma si dimostrano assolutamente inefficaci nei confronti delle forme sessili, risultate resistenti anche alla più alta dose saggiata. Per quel che riguarda il paragone tra i diversi terreni utilizzati, la preincubazione di BTS2 in YEM determinava una produzione di biofilm significativamente inferiore rispetto a quella prodotta in MHB: sarebbe stato quindi logico attendersi che anche il livello di protezione nei confronti degli antibiotici fosse generalmente inferiore. Risultati preliminari hanno indicato però che questo è vero solo in parte: nel caso di cefepime e piperacillina le BIC ottenute nelle due condizioni sono risultate comparabili, suggerendo che il livello di protezione non dipenda esclusivamente dalla quantità di biofilm presente.

\section{DISCUSSIONE}

L'osservazione che BTS2 presenti un incremento della resistenza delle forme sessili rispetto a quelle planctoniche nei confronti di alcuni degli antibiotici saggiati avvalora l'ipotesi che la matrice del biofilm costituisca una barriera efficace contro il passaggio di alcuni antibiotici e dimostra l'importanza di utilizzare test adeguati per valutare l'efficacia della terapia nei pazienti FC. Tuttavia, i nostri dati mostrano che non sempre la capacità di un ceppo di produrre biofilm è sufficiente ad aumentare la sua protezione nei confronti degli antibiotici.

Inoltre l'osservazione che uno stesso ceppo possa variare significativamente la propria capacità di produrre biofilm a seconda del terreno di coltura, dimostra l'importanza di standardizzare questo aspetto, soprattutto perché i risultati preliminari ottenuti utilizzando diversi terreni suggeriscono che le differenze a carico della matrice del biofilm possano essere non solo quantitative ma anche qualitative e che tali differenze possano influire sul livello di protezione conferito nei confronti dei farmaci antimicrobici.

Tabella I. Sensibilità agli antibiotici delle forme planctoniche e delle forme sessili di BTS2 in MHB

\begin{tabular}{|c|c|c|c|c|}
\hline \multirow{3}{*}{ ANTIMICROBICI } & \multicolumn{4}{|c|}{$\begin{array}{c}\text { MIC e BIC }(\mu \mathrm{g} / \mathrm{ml}) \\
\text { Pre-incubazione in } M H B\end{array}$} \\
\hline & \multicolumn{2}{|c|}{ BTS2-00 } & \multicolumn{2}{|c|}{ BTS2-06 } \\
\hline & $\overline{M I C}$ & $\mathrm{BIC}$ & MIC & $\mathrm{BIC}$ \\
\hline Imipenem & 16 & 32 & 32 & 32 \\
\hline Meropenem & 4 & 4 & 4 & 2 \\
\hline Ceftazidime & 2 & 4 & I & 0,5 \\
\hline Cefepime & 8 & $>256$ & 8 & $>256$ \\
\hline Piperacillina & 16 & $>256$ & 4 & $>256$ \\
\hline Aztreonam & 16 & $>256$ & 16 & $>256$ \\
\hline Levofloxacina & 4 & 4 & 16 & 16 \\
\hline Ciprofloxacina & 4 & 2 & 16 & 16 \\
\hline Doxyciclina & 32 & 128 & 32 & 128 \\
\hline SMZ/TM* (5:I) & 2 & 64 & 32 & 128 \\
\hline
\end{tabular}

* Sulfametoxazolo/Trimetoprim 


\section{BIBLIOGRAFIA}

1. Caraher E, Reynolds G, Murphy P, McClean S, Callaghan M. Comparison of antibiotic susceptibility of Burkholderia cepacia complex organisms when grown planktonically or as biofilm in vitro. Eur J Clin Micorbiol Infect Dis 2006; 26: 213-6.

2. Ceri H, Olson ME, Stremick C, Read RR, Morck D, Buret A. The Calgary Biofilm Device: new technology for rapid determination of antibiotic susceptibilities of bacterial biofilms. J Clin Microbiol 1999; 37: 1771-6.

3. M7-A8 Methods for Dilution Antimicrobial Susceptibility Tests for Bacteria That Grow Aerobically (2008).
4. Moskowitz SM, Foster JM, Emerson J, Burns JL. Clinically feasible biofilm susceptibility assay for isolates of Pseudomonas aeruginosa from patients with Cystic Fibrosis. J Clin Microbiol 2004; 42(5): 1915 22.

5. Peeters E, Nelis HJ, Coenye T. Comparison of multiple methods for quantification of microbial biofilms grown in microtiter plates. J Microbiol Methods 2008; 72: $157-65$.

6. Stepanović S, Vuković D, Hola V, et al. Quantification of biofilm in microtiter plates: overview of testing conditions and practical recommendations for assessment of biofilm production by staphylococci. APMIS 2007; 115(8): 891-9. 